\title{
A Secure Audio Teleconference System
}

\author{
D. G. Steer, L. Strawczynski, \\ W. Diffie, M. Wiener \\ BNR, \\ P.O. Box 3511, Station C, Ottawa, Canada \\ K1Y 4 H7.
}

\begin{abstract}
Users of large communication networks often require a multi-party teleconferencing facility. The most common technique for providing secure audio teleconferencing requires the speech of each participant to be returned to clear form in a bridge circuit where it is combined with the speech of the other participants. The combined signal is then re-encrypted for distribution to the conferees. This introduces a security weakness as the bridge works with the clear speech and the cipher keys for all of the participants. In this paper we describe secure conferencing systems in which some of the bridge functions are distributed among the users so that no additional security weakness is introduced. The network conference bridge knows the addresses of the participants and receives and distributes the encrypted speech without modification. The conference system can be used with a number of encryption algorithms and the system is suitable for deployment on digital networks such as ISDN. High quality and robust secure voice communications can be provided with this technique.
\end{abstract}

\section{Introduction}

As the use of secure communication techniques becomes more widespread, the need will grow for convenient secure conferencing facilities. Conferences allow a number of separated callers to participate in a group discussion. Some facilities also include video, data, and graphics facilities for these conferences. To provide a secure conference call, the conference facility is augmented with security features. The intent is to provide privacy (through encryption) and authentication of the the participants.

In this paper we will outline some practical considerations for providing secure audio conference facilities within the public switched telephone network (PSTN). The basic problem to be resolved is to find a practical means to apply the mechanisms of encryption and authentication protocols to a multi-party conference without compromise of the security but within the constraints of the network. The goal is to achieve a conference facility that can be operated by the network supplier without concern for security features and thus leave the customers free to select the security systems to meet their individual needs.

Firstly, we will outline the requirements for a conference facility and point out the limitations of certain implementation techniques. The principal concerns are the non-linear coding of the speech in the PSTN and the requirements for limited bandwidth and minimum delay. We will introduce the concept of a distributed conference system and will review a distributed authentication scheme. A further conferencing technique, suitable for networks with long transmission delays, will also be outlined. We will conclude that secure conference systems are practical in the PSTN, and that they can be safely provided to meet the user's requirements. The Integrated Services Digital Network (ISDN) is especially well adapted to providing these secure services.

\section{Basics of Audio Conferencing}

Figure 1 shows the typical configuration of a conference system and in this section we will review some of the basic operations. This section is included to provide background on the operation of conference circuits. The users (conferees) are connected by the communications network (usually the PSTN) to a "conference bridge" circuit. This 
circuit functions to sum together the speech from all the participants and to distribute this sum (which is the conference signal) to all the conferees. The network connections may be established with the assistance of a conference operator (who may be a participant in the conference) or it may be a "dial-in" conference where participants dial a special directory number to reach the bridge and be included in the conference. Usually an audible signal or a verbal protocol is used to announce when people enter or leave the conference.

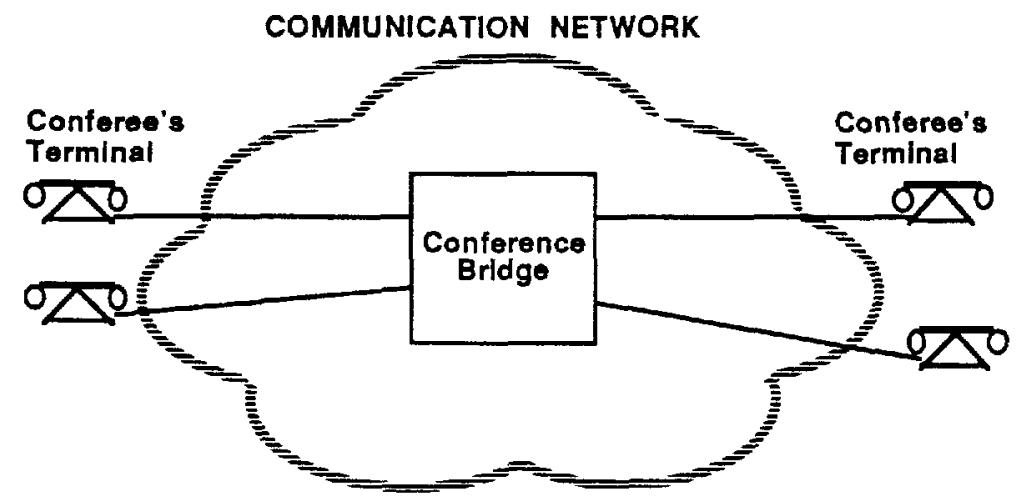

FIGURE 1 - AUDIO CONFERENCE BRIDGE

In concept, the bridge circuit simply sums the speech signals from each participant and distributes this as the conference signal for all to hear. In practice the process is a bit more complicated. One complication is that the signal sent to a participant should not include the participant's own speech. If it does, then transmission delays can cause objectionable echos. Thus the conference sum sent to speakers differs from that sent to passive listeners.

The accumulation of noise is another important consideration in an analogue network. In order to avoid the buildup of noise, the bridge will usually monitor the incoming signals and only distribute the loudest, or perhaps a weighted sum of the 2 loudest, speakers to all the others (see for example [1]). As an alternative, the bridge may include a threshold, and only include a conferee's signal in the conference if the level exceeds the threshold. Noise from the idle channels is kept to a minimum with this arrangement.

Many bridges also include an automatic gain control (AGC) function to equalize the speech volumes of distant and near conferees and of loud and soft speakers.

It is important to keep in mind that in a digital system voice signals are encoded with non-linear codes designed to increase the dynamic range and minimize the effects of quantization noise. The standard PTSN codes for speech are called $\mu$-law (in North America) and A-law (in Europe) [2]. Speech signals encoded with these codes must be converted to a linear representation before the bridge can process them.

There are thus a number of functions required of the bridge to support conferencing:

- summation of speech signals

- code conversions ( $\mu$-law or A-law to linear)

- automatic gain control (AGC)

- identification

The concept of a bridge circuit can be extended to a secure conference as shown in Figure 2. Since the speech signals are now encrypted the bridge circuit can not simply sum the signals as the encryption is typically a non-linear process. (Here we define "nonlinear" to mean that two encrypted signals cannot be summed to yield a third signal that can be decrypted to meaningful speech.) 
Figure 2 shows one possible arrangement for a secure conference. In this case the telephone sets have been augmented with encryption and authentication devices. In normal two-party use these sets provide end-to-end encryption protection. The bridge is also fitted with like devices at its ports. Thus the bridge can receive a secure call from each participant, decrypt the speech, perform the conference operations (linearize, threshold, summation), and then encrypt the result for distribution to the conferees. This is a simple extension to the concept of a conference bridge.

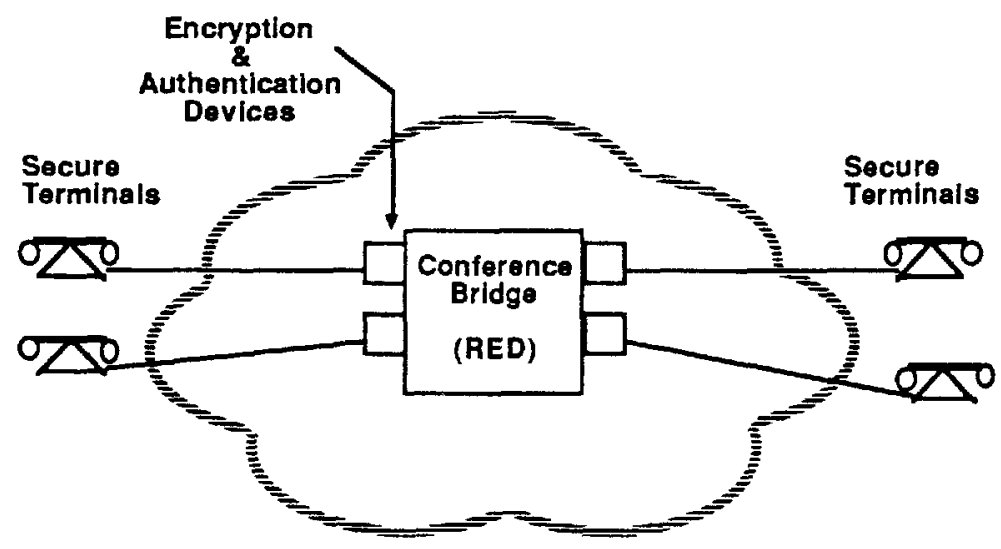

Figure 2 - Conventional Conference Bridge with Security

This method requires the bridge circuit to know the encryption keys for all parties and clear speech is contained within the unit. Thus, the conference bridge must be included when determining the security of the communications system. This is often referred to as a "red" bridge because its internal signals are unprotected by encryption, and it introduces a point of weakness in the system. Some users may not wish to trust the security of a bridge operating outside their direct control. It is thus desirable to make a conference unit that can operate without requiring the speech to be in clear form. This would be referred to as a "black" bridge because all of its signals are protected by encryption.

One possible approach to providing a black bridge has been provided by Brickell, Lee, and Yacobi [3]. In this method, an encryption process is used with certain linear properties which allow the bridge circuit to sum and distribute the signals without the need for decryption. Unfortunately this limits the number of applicable encryption techniques and not all users would be willing to trust these schemes. This method also restricts the speech coding techniques allowed, produces bandwidth expansion, and requires synchronization (in time) of the signals from all of the conferees. This scheme, for example, is unable to use the standard $\mu$-law coded speech common in the PSTN. It is thus desirable to design a method which can function independently of the encryption process and the speech coding, and also does not require any special synchronization.

\section{The Distributed Bridge}

Returning for a moment to Figure 2, note that one place where speech must be in clear form is at the users' telephone terminals. Decrypted speech must be provided at these points for the conferees to hear. The security weakness inherent in the red conference bridge will be eliminated if the conference operation is migrated out to the telephone sets where clear speech must always be available for the users.

One way to accomplish this is shown in Figure 3. In this case each telephone set is equipped with a simple three-party conference circuit in addition to two encryption/decryption units. The sets are connected to the digital switching network by two logical circuits. These access connections would typically be made using digital loop 
technology. A chain-like connection pattern is established between the parties to form the conference. Each party receives the signals from its neighbours, sums these with its local speech input, and forwards the result along the chain. Each party hears the speech of all the others as the signal is passed down the chain. There is no centralized bridge. The function is distributed among the participants. No additional security risk is introduced by this distributed bridge as clear speech must be available at each phone for the conferees to hear anyway. Modern signal processing chips can be used to perform the bridge functions in each user's terminal.

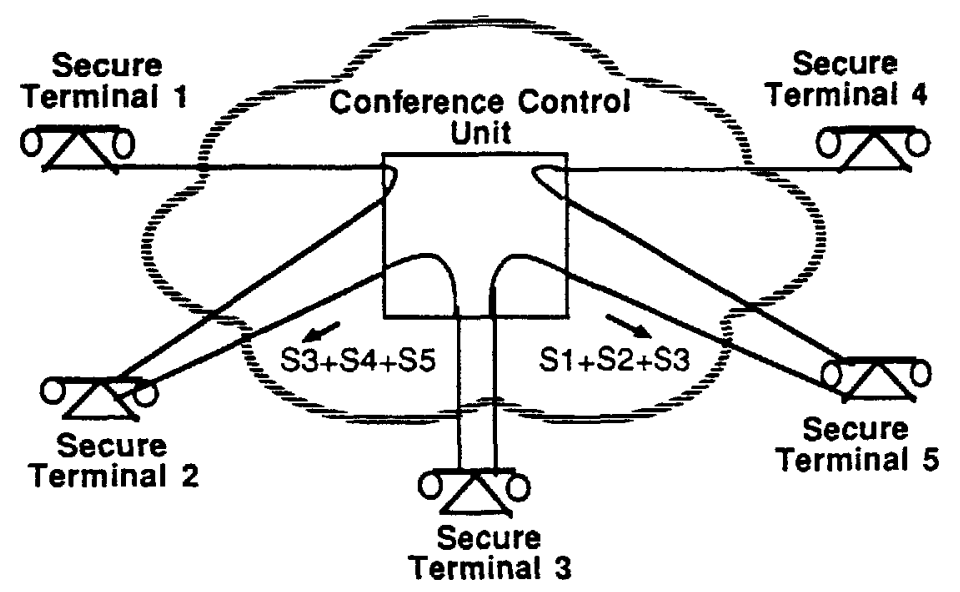

\section{Figure 3 - Distributed Secure Conference Bridge}

One of the difficulties with a chain-like conference connection of this type is that anyone dropping out of the conference breaks the chain. The conference would thus be split into two disconnected parts. This may be overcome by incorporating a conference control unit (CCU) in the network. This CCU keeps the list of network addresses of the (active) participants and is responsible for maintaining the connections through the communications network to all the parties, and automatically reconfiguring the connections when the list of participants changes. This is illustrated in Figure 3 by the rectangle labeled conference control unit. In normal operation, the CCU is under the direction of a conference operator. This may be a network resource person, or it may be simply a participant acting as chairperson and controlling the CCU via a separate communications channel. If parties wish to be added or deleted from the conference, the CCU will automatically reconnect the network to the new configuration. Thus, while we technically have a chain-like connection, operationally it behaves like the common star topology for conference systems. Note that this conference control unit could be implemented as a software program operating in the switching nodes of the communications network.

Figure 3 shows two connections from each phone to the network (except for the ends of the chain). While this could be implemented as two physical connections, modern digital techniques allow the multiplexing of two speech channels on a single pair of wires. The Integrated Services Digital Network (ISDN), for example, directly supports two 64 kbps speech channels (the " $B$ " channels) plus a signaling channel (the " $D$ " channel) on a single subscriber pair. Thus the distributed conference configuration could easily be implemented in an ISDN environment, and in fact we have tested such an arrangement on the BNR ISDN facilities. Other multiplexing techniques can also be used to maintain a single subscriber access connection and to reduce the number of network connections.

In analogue communication networks, the chain-like connection of Figure 3 would be impractical due to the accumulation of transmission and idle channel noise. (Each party receives the accumulated sum of all the noise sources along the chain.) In digital 
transmission, noise does not accumulate on tandem connections, and bit errors can be corrected by the use of suitable error correcting codes. Inherent in any digital speech coding system is quantization and coding noise. However, with suitable speech coding techniques, such as the standard companded PCM, ADPCM [5], and the CCITT wideband audio standard [6], this noise will not accumulate in the tandem connections used in this conference arrangement. The conferencing algorithm at each terminal can be designed with a local speech detector and AGC function. The speech detector would only include local speech input when speech is present to eliminate the accumulation of idle channel noise. The ACG function would equalize the conference levels.

With this technique there is no central bridge to be cracked to compromise security, and the conferees are free to use encryption techniques of their own choosing. The telecommunications supplier can provide the conference control unit to facilitate the service.

\section{Distributing Conference Bridge}

We have described in the previous section a distributed conference bridge in which the parties are connected in a chain-like manner and the conference operations are distributed among the participants. This system is suitable for many applications where the transmission delays are not severe. With this arrangement the coding delays are negligible and the CCU can make the network connections to minimize the transmission delays. However if several satellite connections were involved in the conference the chain-like arrangement would not be satisfactory as significant delay would accumulate along the chain. Figure 4 illustrates the concept of another conferencing arrangement which is not as sensitive to delay.

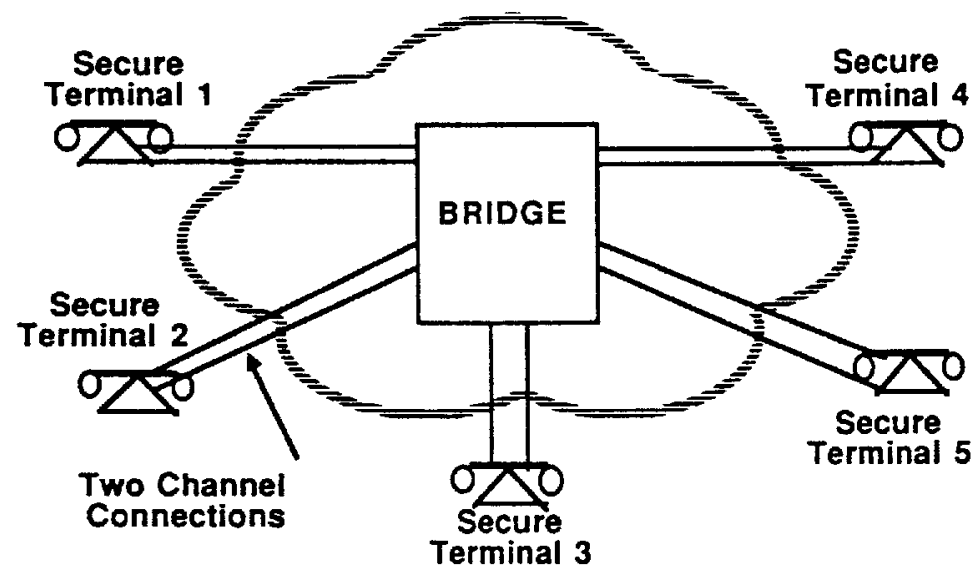

Figure 4 - Distributing Bridge

In this arrangement, each secure telephone set is connected to the PSTN with 2 channels. The use of multiplexing and digital access technology makes this arrangement practical with a single wire pair. Participants in the conference are connected by the network to the distributing bridge. During operation, each terminal sends to the bridge two signals. One of these signals is the encrypted speech from the participant. The second signal is information giving the average volume level of the speech for the preceding time period of say, 4 milliseconds. The bridge unit would examine the volume level information from all the participants, and distribute to each conferee the two loudest encrypted speech signals (other than the conferee's own signal) using the two channel connections. The terminals would receive these two encrypted speech signals, decrypt them and present the sum to the local listener. As the speaker activity in the conference changed, the volume level information would reflect the new speakers, and the bridge would distribute the new 
speakers. Initially, cryptographic session keys would be developed among all participants by means of a protocol such as the one described in the next section section.

In Figure 5 we give a simple illustration of how this might be implemented. Three terminals are involved in this example conference. The diagram illustrates the signals sent by terminals 1 and 2 towards the bridge. These are the encrypted speech signals and the volume level information. In this example, speaker 1 is followed by speaker 2 . The encryption process uses a cipher-feedback mode of operation. The listener at terminal 3 will receive a short pause to signify the change of speakers and allow a change of cryptokey if required. The beginning of the new speaker's encrypted speech would be preceded by a short period of extra data to allow the encryption process to resynchronize. This synchronization period would be muted by the receiver.

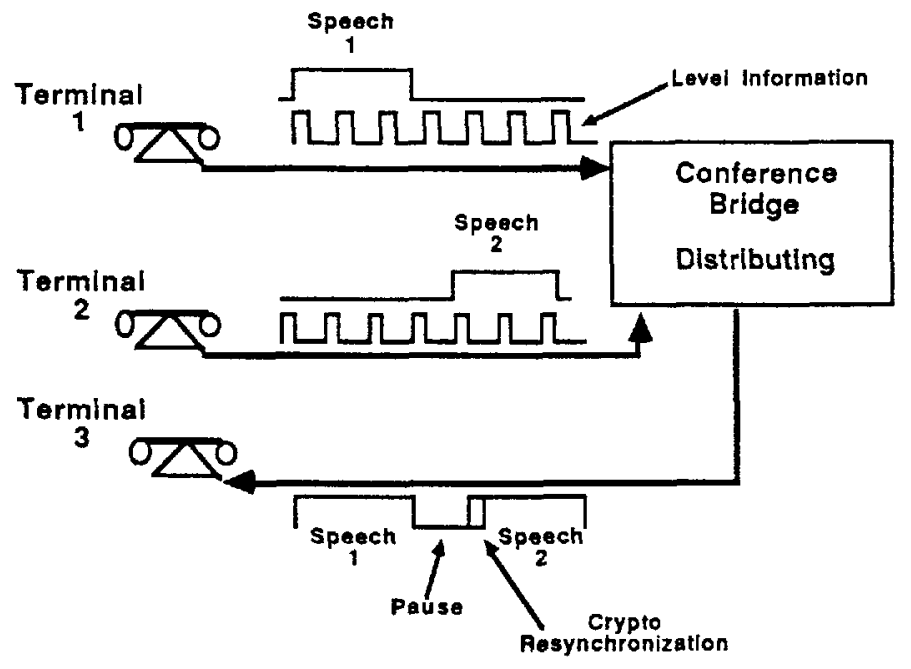

\section{Figure 5 - Operation of Distributing Bridge}

This form of conference bridge handles only encrypted signals and acts only as a distributing center. The users must give out extra information in the form of the average speech level. In the simplest implementation this information would be in the clear. However, it could be sent to the bridge using an encrypted channel. (If the bridge is to remain truly black there is no advantage in doing this.) This average level does not reveal the speech signal but it does represent a (small) leak of information that some users may find unacceptable. This system does not suffer from significant problems with long delay paths as it uses a star topology for the network connections.

\section{Key Distribution and Authentication}

The operation of security features in a large network is dependent upon the proper distribution of encryption keys and the authentication of terminals and users. Practical techniques to provide for two-party end-to-end encryption and authentication systems in the public network are available [4]. These use a "hybrid cryptosystem" with public key techniques being used for key distribution and authentication, and conventional ciphers being used for encryption of the speech. There are two basic requirements. One is for the secure distribution of the conventional encryption keys (also known as session keys), and the second is for the authentication of the participants.

These protocols are designed to provide a new session key for each connection to ensure privacy. These session keys are generated by a random process at each terminal and exchanged between terminals using the exponential key exchange technique of Diffie 
and Hellman [7]. The terminals are identified by means of certificates. These certificates are prepared by the central key management facility (KMF) using public key signature techniques and are unique for each terminal. By exchanging these certificates and verifying the signature of the KMF, the terminals are assured of the identity of their correspondents. Finally to guard against active intruders in the circuit, challenge messages are exchanged between the terminals. Typically the terminal is asked to sign with its private key a message sent by the other terminal. An intruder would be unable to respond to such a challenge with the needed signature. These techniques can be extended to multi-party conference systems and we will briefly outline below a simple key distribution and authentication scheme that is suitable for use with the distributed bridge. Note that while this description is in the context of an audio conference, the protocols are general and could be applied to data or video conferences. Brickell, Lee and Yacobi [3] have also proposed authentication protocols for conference calls. The scheme described below provides for both authentication and the distribution of conventional crypto keys. This provides both privacy and authentication for the conference.

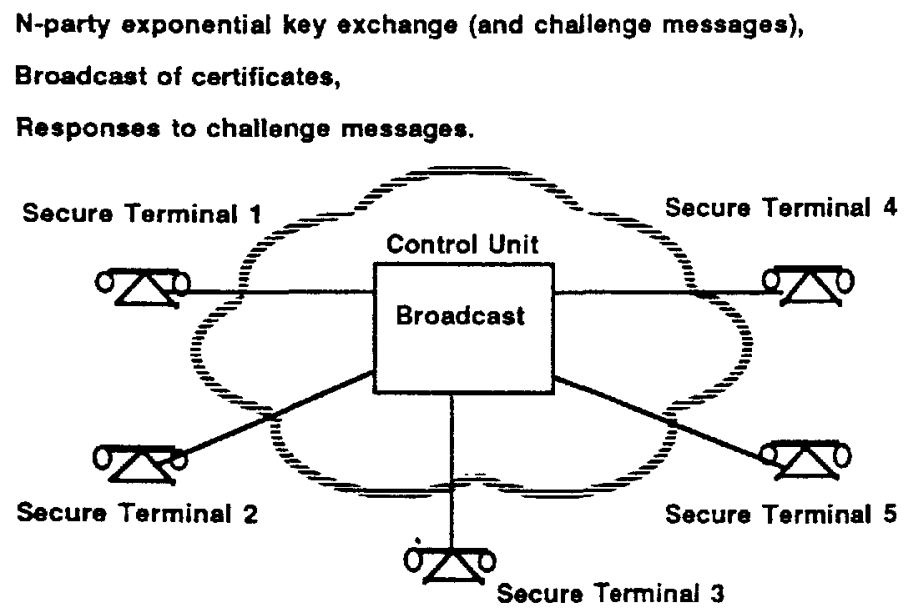

Figure 6 - Distributed Authentication

The key distribution and authentication phase of the conference set up would take place at the beginning of the conference before the onset of speech or data communications. The process would be repeated each time a party is added to the conference. During this set up phase, the conference control unit operates in a broadcast mode so that messages sent to it are distributed to all participants. This is illustrated in Figure 6.

The process for distributed authentication in a conference with $\mathbf{n}$ participants is divided into six steps as follows:

1) Each terminal, $i$, generates a random number $x_{i}$ and calculates the exponential $y_{i} \leftarrow \alpha^{x_{i}}$ and sends this to the CCU for broadcast to the other terminals. ( $\alpha$ is the pre-established common base and calculations are performed modulo a large prime $\mathbf{p}$.)

2) Each terminal now has the set of $y_{i}$. This set can be used to provide an order for the terminals. A convenient order would assign the terminals an index based on the magnitude of the $\mathrm{yi}_{\mathrm{i}}$. (Terminal 1 generated the smallest of the $\mathrm{yi}_{\mathrm{i}}$ etc. In the unlikely event that two of the yi were equal then the terminals would be expected 
to submit new values.) The order could also be established by the CCU based on the chain topology of the connections.

3) Each of the terminals will now calculate an exponential of the following form :

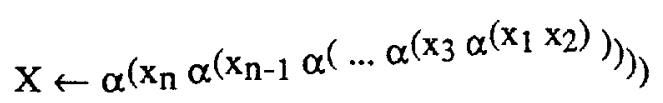

Terminals 1 and 2 can calculate $X$ based on their knowledge of the $y_{i}$ and their own $x_{i}$. However, terminals 3 to $n$ require further information to calculate $X$. This information comes in the form of the numbers $w_{1} \ldots w_{n-1}$ calculated by the terminals and broadcast to the other terminals. The terminals proceed as follows :

Terminal 1 performs the following operations :

$\mathrm{X} \leftarrow \mathrm{x}_{1}$,

for $\mathrm{j}=2$ to $\mathrm{n}$ do the following :

$$
\mathrm{X} \leftarrow \mathrm{yj}_{\mathrm{j}} \mathrm{X} \text {. }
$$

Terminal $\mathrm{i}$ (for $\mathrm{i}=\mathbf{2}$ to $\mathbf{n} \mathbf{- 1}$ ) performs the following : receive $w_{i}-1$ via the $C C U$

$$
\begin{aligned}
& \left.\quad \text { (Terminal } 2 \text { uses } \mathrm{w}_{1} \leftarrow \mathrm{y}_{1}\right), \\
& \mathrm{X} \leftarrow \mathrm{w}_{\mathrm{i}-1} \mathrm{x}_{\mathrm{i}}, \\
& \mathrm{w}_{\mathrm{i}} \leftarrow \alpha^{\mathrm{X}},
\end{aligned}
$$

broadcast $w_{\mathrm{i}}$ to all other terminals via the $\mathrm{CCU}$, for $\mathrm{j}=\mathrm{i}+1$ to $\mathrm{n}$ do the following :

$$
\mathrm{X} \leftarrow \mathrm{yj}_{\mathrm{j}} \mathrm{X} \text {. }
$$

Terminal $\mathbf{n}$ performs the following operation :

$$
\mathrm{X} \leftarrow \mathrm{w}_{\mathrm{n}-1} \mathrm{x}_{\mathrm{n}}
$$

4) All terminals now share the number $X$ which can be used to derive a common session key for the conference. This key would be used to encrypt all further transmissions. It is not necessary to use a common session key. The number $X$ could also be used by pairs of terminals to form a unique session key for their connections.

5) Each terminal broadcasts (via the CCU) its certificate, and verifies the signature on all received certificates.

6) Each terminal signs with its secret key a suitable hash function of the set of numbers $\mathrm{y}_{i}$ and broadcasts this (via the $\mathrm{CCU}$ ) to all other terminals. Each terminal verifies the signature of these messages.

Step 1 requires a total of $\mathbf{n}$ broadcast messages. Step 3 requires $\mathbf{n}-\mathbf{2}$ messages. Steps 5 and 6 each require $n$ broadcast messages. This gives a total of of 4 n-2 broadcast messages. Note, however, that the certificates and response messages ( 5 and 6 ) could be combined into a single message for transmission. In this case the total number of broadcast messages is $3 n-2$. The number of exponentiation operations to be performed by each terminal for key generation depends on the terminal's location in the sequence. Terminals 1 and 2 must perform $\mathbf{n}$ exponentiations. Verifying the certificates, and 
responding to the challenge for authentication would require an additional 2 operations and thus a total of $3 \mathbf{n}$ exponentiations could be required. Note that this grows linearly with $\mathbf{n}$. Once the authentication phase is completed, the conference control unit can switch from the broadcast to the chain mode. With this procedure, the authentication process is distributed and thus no party need rely on a centralized bridge for the security of the call. However, a trusted key management facility is required for the creation of the user's certificates.

The procedure for adding or deleting parties to the conference will depend on the security policy of the participants. Some policies may require a new session key and a complete re-authentication of all parties each time the network configuration changes. In the simple scheme described above, if a common session key is used, the chain may be reconnected (with an appropriate mechanism for crypto resynchronization) when a party leaves. An additional party may be accomodated by simply labeling it as terminal $n+1$ and appending its input $\left(\mathrm{y}_{\mathrm{n}+1}\right)$ to the accumulated chain calculation. This would result in a new common number $X^{\prime}$ and a new session key. Changes in the participation in the conference can thus be accomodated with a minimum of interruption.

Thus it is practical to provide a true black bridge for secure teleconferencing in the public or other large networks. A wide variety of digital encryption processes can be used. The authentication mechanisms can function with many public key systems. Users are free to choose whatever system they feel comfortable with. They are also free to change the operation of the security system without consultation with the network provider. These are significant benefits for the widespread use of and confidence in the system.

\section{Conclusions}

We have described secure teleconferencing systems which can be operated in the public communications network. In these systems, advantage is taken of the fact that clear speech must be available at each telephone set for the local participant, and this can be used with a distributed conference circuit to provide a multi-party conferencing system. The network conference control unit knows the addresses of all the participants but its role is limited to providing channel connections and demultiplexing and distributing unaltered signals from the participants. The conference unit does not need to know any security information. The system can operate with a number of security methods and network configurations, and the users are free to select the techniques that they are most comfortable with. The system is suitable for implementation in digital networks such as ISDN, and can provide extremely high quality and robust operation.

\section{References}

[1] John Ellis, Bruce Townsend; State of the art in Teleconferencing. Telesis 1987 one pp23-31.

[2] David R. Smith; Digital Transmission Systems.

Van Nostrand Reinhold Co., New York. 1985 pp77-88.

[3] E.F. Brickell, P,J, Lee, Y. Yacobi; Secure Audio Teleconference. Advances in Cryptology - Crypto'87 (Proceedings), pp418-426. Carl Pomerance (Ed.), SpringerVerlag, Berlin Heidelberg, 1988.

[4] W. Diffie, B. O'Higgins, L. Strawczynski, D.G. Steer, Secure Communications with the Integrated Services Digital Network (ISDN). Proc 3rd Annual Symposium on Physical/Electronic Security, Philadelphia Chapter AFCEA pp 34.1-34.5, August 1987.

[5] CCITT Recommendation G.721 (ADPCM).

[6] P. Mermelstein; G.722, A new CCITT Coding Standard for Digital Transmission of Wideband Audio Signals. IEEE Communications, Volume 26, No 1, pp 8-15 Jan 1988.

[7] W.Diffie, M.Hellman, New Directions in Cryptography. IEEE Trans Information

Theory IT-22:pp 644-654, 1976. 